\title{
Comment on "Limited cardiopulmonary capacity in patients with liver cirrhosis when compared to healthy subjects"
}

\author{
Mao Sun ${ }^{\oplus}$, Bin Zhao ${ }^{\circledR}$, Bingchuang Huang ${ }^{2} \odot$, Yanchun $\mathrm{Mou}^{3 *} \odot$
}

Dear Editor,

We have perused with interest your article entitled "Limited cardiopulmonary capacity in patients with liver cirrhosis when compared to healthy subjects" in which Nasser et al. ${ }^{1}$ found that liver cirrhosis can compromise the quality of life of patients, mainly by inducing metabolic alterations, which can impair functional capacity and lead to a sedentary lifestyle. However, a number of issues were raised from our point of view.

So far, we do not know how to select patients with liver cirrhosis and healthy subjects for this study. For the chronic liver disease-matched analysis, for which three groups were required, control patients with liver disease were matched with the patients with liver cirrhosis and control patients without liver disease were matched with the patients with liver disease.
How to define inactive lifestyle, consisting of daily routine activities and never having been engaged in exercise programs for a long time? The study should provide a means for quantitative measurement of inactive lifestyle. Physical activity level could be evaluated by the Physical Activity Scale. The references for the evaluation of physical activity should be given in this study.

\section{AUTHORS" CONTRIBUTION}

MS: Writing - original draft, Writing - review \& editing. BZ: Data curation, Formal analysis, Writing - original draft. BH: Data curation, Formal analysis, Writing - original draft. YM: Conceptualization, Writing - review \& editing.

\section{REFERENCE}

1. Nasser I, Miranda H, Perez RM, Cabiddu R, Malosa L, Dias I, et al. Limited cardiopulmonary capacity in patients with liver cirrhosis when compared to healthy subjects. Rev Assoc Med Bras (1992). 2021;67(1):94-100. https://doi.org/10.1590/1806-9282.67.01.20200449

\footnotetext{
1970 Hospital, Department of Hepatobiliary General Surgery - Weihai, China.

2970 Hospital, Department of Hepatobiliary Surgery - Weihai, China.

3970 Hospital, Department of Hyperbaric Oxygen - Weihai, China.

*Corresponding author: muyanchun404@163.com

Conflicts of interest: the authors declare there is no conflicts of interest. Funding: none.

Received on August 13, 2021. Accepted on August 29, 2021.
} 